\title{
Enterovirus A71 and coxsackievirus A16 show different replication kinetics in human neuronal and non-neuronal cell lines
}

\author{
Thinesshwary Yogarajah ${ }^{1} \cdot$ Kien Chai Ong ${ }^{2} \cdot$ David Perera $^{3} \cdot$ Kum Thong Wong $^{1}$
}

Received: 9 September 2016/ Accepted: 19 October 2016

(C) Springer-Verlag Wien 2016

\begin{abstract}
Enterovirus A71 (EV-A71) and coxsackievirus A16 (CV-A16) are closely related enteroviruses that cause hand, foot and mouth disease (HFMD) in children. Serious neurological complications almost always occur in EVA71 infection, but are rare in CV-A16 infection. Based on the hypothesis that this may be because EV-A71 infects neuronal cells more easily than CV-A16, we compared virus infection, replication and spread of EV-A71 and CVA16 in SK-N-SH cells. We found that CV-A16 invariably showed significantly lower replication and caused less necrotic cell death in SK-N-SH cells, compared with EVA71. This was not due to a lower proportion of CV-A16infected cells, since both viruses showed similar proportions of infected cells at all time points analyzed. Furthermore, reduced replication of CV-A16 in SK-N-SH cells does not appear to be due to limited viral receptor availability, which might limit viral entry, because experiments with viral RNA-transfected cells showed the same results as for live virus infections. On the other hand, no
\end{abstract}

Kum Thong Wong

wongkt@ummc.edu.my

Thinesshwary Yogarajah

thiki36@yahoo.com

Kien Chai Ong

kcong@ummc.edu.my

David Perera

davidperera@yahoo.com

1 Department of Pathology, Faculty of Medicine, University of Malaya, 50603 Kuala Lumpur, Malaysia

2 Departments of Biomedical Science, Faculty of Medicine, University of Malaya, Kuala Lumpur, Malaysia

3 Institute of Health and Community Medicine, University Malaysia Sarawak, Sarawak, Malaysia differences were observed between EV-A71 and CV-A16 in RD cells and results were generally similar in RD cells for both viruses. Taken together, our findings suggest that the poor growth of CV-A16 and EV-A71in SK-N-SH cells, compared with RD cells, may be due to cell type-specific restrictions on viral replication and spread. Furthermore, the lower viral replication and necrotic cell death in $\mathrm{CV}$ A16-infected SK-N-SH cells, compared with EV-A71-infected SK-N-SH cells, is consistent with the lower prevalence of neurotropism observed in CV-A16-associated HFMD outbreaks. Nonetheless, in vivo data and more extensive comparisons of different viral strains are essential to confirm our findings.

\section{Introduction}

Enterovirus 71 (EV-A71) and coxsackievirus A16 (CVA16) are human enteroviruses belonging to the species Enterovirus A, genus Enterovirus, within the Picornaviridae family. Both viruses are $\sim 30 \mathrm{~nm}$, each containing a single-stranded, positive-sense RNA genome of approximately $7.5 \mathrm{kbp}$ and they share about $80 \%$ of sequence similarity [65]. Furthermore, they use the same Scavenger receptor class $\mathrm{B}$, member 2 (SCARB2) as a receptor for cell entry and possibly other attachment receptors, such as P-selectin glycoprotein ligand-1 (PSGL-1) and heparin sulphate [49, 60-62]. EV-A71 and CV-A16 are known etiological agents for sporadic and epidemic hand, foot and mouth disease (HFMD), a common infectious disease frequently seen in young children aged 5 and below $[44,53,54]$. In recent years, large outbreaks of HFMD have been mainly reported in the Asia-Pacific region [1, 18, 19, 21, 32-34]. HFMD is characterized by lesions on the skin of the hand, foot, buttock and oral mucosa [25]. 